\title{
Impact of xerostomia on dysphagia after chemotherapy-intensity-modulated radiotherapy for oropharyngeal cancer: Prospective longitudinal study
}

\author{
Jeffrey M. Vainshtein, MD, ${ }^{1 \dagger}$ Stuart Samuels, MD, PhD, ${ }^{1}$ Yebin Tao, MSc, ${ }^{2}$ Teresa Lyden, MA, CCC-SLP, ${ }^{3}$ Marc Haxer, MA, CCC-SLP, ${ }^{3}$ \\ Matthew Spector, MD, ${ }^{4}$ Matthew Schipper, PhD, ${ }^{1,2}$ Avraham Eisbruch, $\mathrm{MD}^{1 *}$ \\ ${ }^{1}$ Department of Radiation Oncology, University of Michigan, Ann Arbor, Michigan, ${ }^{2}$ Department of Biostatistics, University of Michigan, Ann Arbor, Michigan, ${ }^{3}$ Department of \\ Speech Pathology, University of Michigan, Ann Arbor, Michigan, ${ }^{4}$ Department of Otolaryngology, University of Michigan, Ann Arbor, Michigan. \\ Accepted 13 September 2015 \\ Published online 25 November 2015 in Wiley Online Library (wileyonlinelibrary.com). D0l 10.1002/hed.24286
}

\begin{abstract}
Background. The purpose of this study was to assess how xerostomia affects dysphagia.

Methods. Prospective longitudinal studies of 93 patients with oropharyngeal cancer treated with definitive chemotherapy-intensity-modulated radiotherapy (IMRT). Observer-rated dysphagia (ORD), patient-reported dysphagia (PRD), and patient-reported xerostomia (PRX) assessment of the swallowing mechanics by videofluoroscopy (videofluoroscopy score), and salivary flow rates, were prospectively assessed from pretherapy through 2 years.

Results. ORD grades $\geq 2$ were rare and therefore not modeled. Of patients with no/mild videofluoroscopy abnormalities, a substantial proportion had PRD that peaked 3 months posttherapy and subsequently improved. Through 2 years, highly significant correlations were observed
\end{abstract}

between PRX and PRD scores for all patients, including those with no/ mild videofluoroscopy abnormalities. Both PRX and videofluoroscopy scores were highly significantly associated with PRD. On multivariate analysis, PRX score was a stronger predictor of PRD than the videofluoroscopy score.

Conclusion. Xerostomia contributes significantly to PRD. Efforts to further decrease xerostomia, in addition to sparing parotid glands, may translate into improvements in PRD. (C) 2015 Wiley Periodicals, Inc. Head Neck 38: E1605-E1612, 2016

KEY WORDS: xerostomia, dysphagia, head neck cancer, patientreported outcomes, intensity-modulated radiotherapy (IMRT)

\section{INTRODUCTION}

Patients with oropharyngeal cancer, the majority of whom have human papillomavirus-related (HPV-positive) oropharyngeal cancer, have excellent oncologic outcomes after chemoradiotherapy (CRT), which makes the prevention of radiation-related toxicities a priority. ${ }^{1}$ Dysphagia is a common sequel of CRT for head and neck cancer and a major determinant of patient-reported quality of life (QOL). ${ }^{2,3}$ Previous studies have demonstrated that CRT can affect the mechanics of swallowing, resulting in increased bolus transit time, decreased movement of the tongue base toward the posterior pharyngeal wall, reduced laryngeal elevation, and food retention in the oral cavity. ${ }^{4,5}$ These changes are uncomfortable and place patients at risk for aspiration-related complications. ${ }^{6}$ Despite aggressive management of dysphagia with rehabilitation

*Corresponding author: A. Eisbruch, Department of Radiation Oncology, University of Michigan Hospital, 1500 E. Medical Center Drive, Ann Arbor Ml 48109. E-mail eisbruch@umich.edu

${ }^{\dagger}$ Current address: Department of Radiation Oncology, Winship Cancer Institute, Emory University School of Medicine, Atlanta, Georgia

Contract grant sponsor: Supported in part by National Institutes of Health (NIH) grant P01 CA59827 and the Newman Family Foundation.

This work was presented at the 56th Annual Meeting of American Society for Radiation Oncology (ASTR0), San Francisco, CA, September 14-16, 2014. and exercise regimens, many patients do not regain their pretreatment swallowing function, and some may require prolonged feeding tubes for nutritional support. ${ }^{4,7}$ Efforts to characterize the functional anatomy of swallowing using videofluoroscopy have identified organs at risk for CRT, including pharyngeal constrictors, glottis, supraglottic larynx, and upper esophagus. ${ }^{5,8,9}$ We have previously reported our institutional experience with definitive organ-sparing CRT for oropharyngeal cancer, demonstrating that dysphagia and its complications are reduced by limiting the dose to the swallowing-related organs using intensity-modulated radiotherapy (IMRT). . $^{3,8,10-12}$

Despite use of swallowing organ-sparing IMRT, many patients still complain of difficulty swallowing dry foods. There is evidence that patient-reported dysphagia (PRD) often does not correlate with objective measures of dysphagia (ie, the feeling of difficulty swallowing may not relate to the dysfunction of the swallowing structures). ${ }^{13-15}$ This disparity implies that other factors related to eating, not involving dysfunction of the swallowing structures, may be responsible for the sensation of dysphagia. Indeed, xerostomia, another common consequence of head and neck radiotherapy (RT), can make swallowing, especially dry food, difficult to the point where patients require excessive water or simply avoid such foods. ${ }^{16,17}$ Although parotid gland-sparing with IMRT in recent years has decreased xerostomia rates, approximately $25 \%$ to 
$50 \%$ of patients still report persistent xerostomia after IMRT. ${ }^{18,19}$ In order to clarify the relationships between xerostomia and dysphagia, we sought to evaluate the correlations among patient-reported xerostomia (PRX), PRD, and functional assessments of swallowing (videofluoroscopy) and salivary output (stimulated salivary flow [SSF] rates) in patients who participated in prospective studies of chemo-IMRT for oropharyngeal cancer.

\section{MATERIALS AND METHODS}

\section{Patients and therapy}

Between May 2003 and March 2011, 93 patients with newly diagnosed stage III to IV oropharyngeal cancer were treated on 2 prospective consecutive institutional review board-approved studies of organ-sparing chemoIMRT for locally advanced oropharyngeal cancer. Patient eligibility and treatment have been previously detailed and were similar in both protocols. ${ }^{8,11,20}$ Seventy-three patients were enrolled on a phase II study of chemoIMRT aiming to reduce dysphagia by sparing the swallowing-related structures, ${ }^{8,11}$ and 20 patients on a subsequent study assessed the dose-effect relationships based on cone-beam CT-derived actually delivered organ doses. $^{20}$ IMRT prescription doses were $70 \mathrm{~Gy}$ to the gross target volume primary and nodal tumor volume; 59 to 63 Gy to high-risk clinical target volumes (CTVs); and 56 to 59 Gy to low-risk CTVs, delivered over 35 daily fractions. Gross target volumes and CTVs were uniformly expanded 3 to $5 \mathrm{~mm}$ to create planning target volumes. Bilateral necks were treated in all patients. IMRT treatment planning was performed with intent to minimize the dose to the parotid glands, contralateral submandibular gland, noninvolved oral cavity, glottic and supraglottic larynx, esophagus, and the pharyngeal constrictor muscles, with dosimetric goals and optimization algorithms, as previously detailed. ${ }^{8}$ The contralateral submandibular glands were preferentially spared in the latter years of the study period using an optimization goal of mean dose $<39$ Gy, based on a previous study. ${ }^{21}$ All patients received concurrent weekly carboplatin (area under the curve $[\mathrm{AUC}]=1)$ and paclitaxel $(30 \mathrm{mg} / \mathrm{m} 2)$.

\section{Patient-reported dysphagia and xerostomia assessments}

Three validated health-related quality of life (HRQOL) instruments were administered to enrolled patients at pretreatment and 3, 6, 12, 18, and 24 months after completion of CRT; these consisted of the Head and Neck Quality of Life (HNQOL) questionnaire, ${ }^{22}$ the University of Washington Quality of Life (UWQOL) questionnaire, ${ }^{2}$ and the Xerostomia Questionnaire (XQ). ${ }^{18}$ PRD was assessed using the swallowing question from the HNQOL (HNQOL-Sw) instrument ("As a result of your head and neck condition or treatment, over the past four weeks, how much have you been bothered by problems with swallowing soft foods and/or solids?") and the UWQOL swallowing (UWQOL-Sw) instrument ("Over the past week, I [1] swallow normally, [2] cannot swallow certain solid foods, [3] can only swallow soft foods, [4] can only swallow liquid foods, [5] cannot swallow"). PRX was assessed using a summary score of the XQ (XQ score), a validated instrument containing 8 questions that assess patients' mouth and throat dryness and difficulty with talking, chewing, swallowing, and sleeping because of dryness. $^{18}$ Four questions assess dryness during eating/ speaking, and 4 questions assess dryness while not eating. Patients rate each question on the XQ from 0 to 10 on an 11-point ordinal Likert scale, in which higher scores indicate greater dryness or discomfort because of dryness. The HRQOL instrument individual question and summary scores were normalized on a linear 100 -point scale, with 0 representing no toxicity or negative QOL effects and 100 representing the worst possible QOL. ${ }^{3}$ Scores of $0,25,50$, 75 , and 100 approximated responses of "none," "mild/ slight," "moderate," "severe," and "extreme," respectively.

\section{Observer-rated dysphagia and xerostomia assessments}

Observer (physician)-rated dysphagia (ORD) was graded at each follow-up visit using the Radiation Therapy Oncology Group/European Organization for Research and Treatment of Cancer scoring system $(0=$ no dysphagia; $1=$ symptomatic, able to eat regular diet; 2 = symptomatic, altered eating/swallowing; 3 = symptomatic, severely altered eating/swallowing requiring feeding tube; and $4=$ complete obstruction or perforation). ${ }^{23}$ Observer (physician)-rated xerostomia scores were not included in the present study given our demonstration in prior studies that observer-rating underestimated xerostomia severity compared with patient-reported scores, and did not correlate with the SSF rates, unlike the significant correlations observed for patient-reported scores. ${ }^{24}$

\section{Functional swallowing assessments by modified barium swallow videofluoroscopy}

Functional assessment of dysphagia was performed by modified barium swallow videofluoroscopy, as previously detailed. $^{3,8}$ Videofluoroscopy was performed pretreatment and at 3, 12, and 24 months posttherapy, with results quantified using the Swallowing Performance Status Scale score: $1=$ normal swallow; 2 = within functional limits; $3=$ mild impairment; $4=$ mild-moderate impairment; $5=$ moderate impairment; $6=$ moderate-severe impairment; and $7=$ severe impairment, ${ }^{25-28}$ as in our prior studies. $3,8,11$

\section{Salivary flow assessments}

Functional assessments of xerostomia were performed by measurements of the salivary output from the major salivary glands, as previously described. ${ }^{18,21}$ Unstimulated and SSF rates were measured selectively for each parotid and submandibular gland at the same timepoints as HRQOL assessments, as previously described. ${ }^{29}$ For analysis purposes, the sum of the SSF output (in $\mathrm{mL} / \mathrm{min}$ ) from all glands was used for all analyses.

\section{Statistical analysis}

Descriptive statistics were used to calculate means and SDs for salivary flow rates and XQ scores at pretreatment and at 3, 6, 12, 18, and 24 months posttherapy. The prevalence of swallowing impairment, as measured by the Swallowing Performance Status Scale score, of PRD, as measured by HNQOL-Sw and UWQOL-Sw question 
TABLE 1. Patient characteristics.

\begin{tabular}{lc}
\hline Characteristics & Statistics \\
\hline Male, no. (\%) & $83(89)$ \\
Age, y, median (range) & $56(40-78)$ \\
Primary tumor site, no. (\%) & \\
Tonsil & $45(48)$ \\
Base of tongue & $48(52)$ \\
T classification, no. (\%) & \\
T1 & $12(13)$ \\
T2 & $40(43)$ \\
T3 & $20(22)$ \\
T4 & $21(23)$ \\
N classification, no. (\%) & \\
N0 & $5(5)$ \\
N1 & $8(9)$ \\
N2 & $70(75)$ \\
N3 & $10(11)$ \\
HPV status, no. (\%) & \\
Positive & $86(93)$ \\
Negative & $1(1)$ \\
N/A & $6(7)$ \\
Smoking history, no. (\%) & \\
Never smoker & $35(38)$ \\
Former smoker & $34(37)$ \\
Current smoker & $24(26)$ \\
Pack-years, median (range) & $7(0-140)$ \\
\hline
\end{tabular}

Abbreviations: HPV, human papillomavirus; N/A, not available.

scores, and of ORD was calculated at pretreatment and 3, 12 , and 24 months posttherapy. The prevalence of PRD by HNQOL-Sw and UWQOL-Sw scores was determined for patients with no or minimal functional swallowing impairment (Swallowing Performance Status Scale score $\leq 3$ ) at pretreatment and 3, 12, and 24 months posttreatment. Spearman rank correlation $(\rho)$ was used to assess the association between XQ score and salivary flow rates, XQ score and HNQOL-Sw and UWQOL-Sw scores, and Swallowing Performance Status Scale score and salivary flow rates at each timepoint for all patients and separately for patients with and without functional swallowing impairment on videofluoroscopy (Swallowing Performance Status Scale scores $\geq 4$ and $\leq 3$, respectively).

We fitted proportional odds models to assess the association between dysphagia and xerostomia, and conducted univariate as well as multivariate analysis with adjustment for covariates. The magnitude of the association was quantified using normalized odds ratios (ORs), calculated as the OR per SD increase in the predictors. A $p$ value $\leq .05$ was accepted as statistically significant. Statistical analysis was conducted using SAS (version 9.4; SAS Institute, Cary, NC).

\section{RESULTS}

Baseline characteristics for the 93 enrolled patients are shown in Table 1 . The majority of patients were men with primary tumors that were HPV-positive (93\%). HRQOL data were available for 92 patients at pretreatment, 91 at 3 months, 80 at 12 months, and 70 at 24 months. Videofluoroscopy and salivary flow data were available, respectively, for 92 and 87 patients at pretreat- ment, 87 and 79 at 3 months, 81 and 73 at 12 months, and 65 and 61 at 24 months.

\section{Salivary flow rates and patient-reported xerostomia}

Mean SSF rates were $0.87 \mathrm{~mL} / \mathrm{min}( \pm \mathrm{SD} 0.72)$ pretherapy, declined to $0.19 \mathrm{~mL} / \mathrm{min}( \pm 0.35)$ at 3 months, and thereafter improved to 0.29 ( \pm 0.38$), 0.32$ ( \pm 0.33$), 0.39$ $( \pm 0.42)$, and $0.37( \pm 0.38) \mathrm{mL} / \mathrm{min}$ at $6,12,18$, and 24 months posttherapy, respectively. Mean XQ scores pretherapy were $6.5( \pm \operatorname{SD} 13)$, worsened to $51( \pm 23)$ and $46( \pm 24)$ at 3 and 6 months, and thereafter improved to $37( \pm 24), 34( \pm 23)$, and $31( \pm 22)$ at 12,18 , and 24 months, respectively.

Assessment of the correlations between SSF rates and the XQ scores revealed no significant correlation pretherapy, but statistically significant correlations $(p<.05)$ at each timepoint posttherapy. Spearman's correlation coefficients were only modest, however: $-0.37,-0.35$, $-0.55,-0.38$, and -0.35 at $3,6,12,18$, and 24 months, respectively (Table 2); the negative coefficients denote increased (worsened) XQ scores as salivary output reduced.

\section{Observer-rated dysphagia}

ORD grade $\geq 2$ was infrequent. Grades 2 and 3 dysphagia were observed in $7 \%$ and $4 \%$ of patients at 6 months, $2.7 \%$ and $2 \%$ at 12 months, and $1 \%$ and $1 \%$ at both 18 and 24 months, respectively. Because of the very low frequency of grades $\geq 2$ ORD at 12 months and beyond, ORD was not analyzed further.

\section{Functional swallowing assessment by videofluoroscopy and patient-reported dysphagia}

Functional swallowing impairment by Swallowing Performance Status Scale score and PRD by HNQOL-Sw and UWQOL-Sw score at each timepoint are shown in Figure 1. Pretherapy Swallowing Performance Status Scale scores were mostly within functional limits, whereas at 3 months most scores showed mild-tomoderate impairment, with $14 \%$ showing severe impairment. By 12 and 24 months the rate of patients "within functional limits" increased to $25 \%$ and $32 \%$, respectively, and "severe impairment" was rare. A similar pattern over time was observed in the HNQOL-Sw and UWQOL-Sw scores (see Figure 1).

\section{Patient-reported dysphagia in patients without functional swallowing impairment}

We next sought to evaluate whether patients without functional swallowing impairment on videofluoroscopy may nonetheless perceive the feeling of dysphagia (Table 3). Of 39 patients with either no or mild impairment on videofluoroscopy (Swallowing Performance Status Scale score $\leq 3$ ) at 3 months, $21 \%$ reported at least moderate swallowing-related bother on the HNQOL-Sw assessment. On the UWQOL-Sw assessment, 59\% of patients with Swallowing Performance Status Scale score $\leq 3$ reported inability to swallow certain solid foods, and $13 \%$ reported swallowing ability limited to only soft foods. At 12 and 24 months, moderate or greater severity swallowing- 

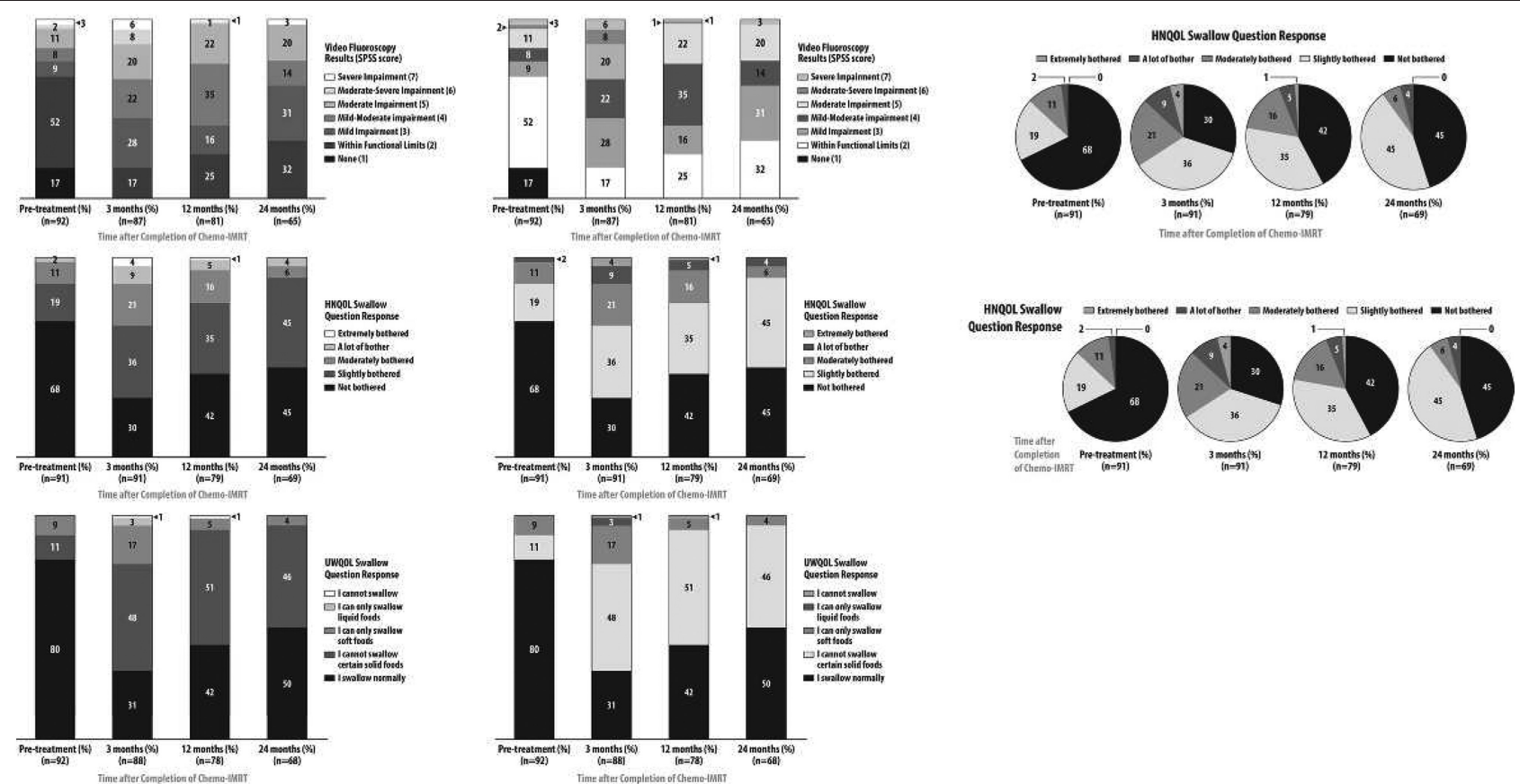

FIGURE 1. Swallowing impairment pretreatment and after chemoradiation as rated by (A) videofluoroscopy assessment, (B) patient selfassessment using the Head and Neck Quality of Life (HNQOL) Instrument Swallowing question, and (C) University of Washington Quality of Life (UWQOL) Instrument Swallowing Question.

related bother was reported on HNQOL-Sw by $12 \%$ and $5 \%$ of patients with Swallowing Performance Status Scale $\leq 3$, respectively. By UWQOL-Sw, $30 \%$ of patients with no/mild videofluoroscopy abnormalities at 12 months reported inability to swallow certain solid foods and $3 \%$ reported being able to swallow only soft foods; the respective rates at 24 months were similar at $28 \%$ and $3 \%$.

\section{Relationship between patient-reported and functional assessments of xerostomia and dysphagia}

Given that a substantial proportion of patients reported swallowing problems despite mild or no swallowing abnormalities on videofluoroscopy, we explored whether the perception of dysphagia may be related to, and potentially attributable to, PRX. At pretreatment, XQ and HNQOL-Sw scores were moderately correlated $(\rho=0.43$; $p<.001)$, reflecting the high proportion of patients $(84 \%)$ who reported either no or slight/mild symptoms (normalized score $\leq 25$ of 100 ) on both the XQ and HNQOL-Sw assessments, consistent with an absence of dysphagia or xerostomia in most patients pretherapy. Posttreatment, the strength of the correlation between XQ and HNQOL-Sw scores increased $(\rho=0.57,0.52$, and 0.54 at 3,12 , and 24 months, respectively; all $p<.001$; Table 2). Similar magnitude correlations were observed between XQ scores and UWQOL-Sw scores $(\rho=0.33,0.47,0.61$, and 0.47 at pretherapy and 3, 12, and 24 months posttreatment, respectively; all $p<.01$; Table 2 ).

In comparison, the correlations between SSF rates and both HNQOL-Sw scores $(\rho=0.014$ and $p=.90$ at pretherapy; $\rho=-0.28$ and $p=.01$ at 3 months; $\rho=-0.30$ and $p=.01$ at 12 months; and $\rho=-0.23$ and $p=.08$ at 24 months) and UWQOL-Sw scores $(\rho=-0.12$ and $p=.27$ at pretherapy; $\rho=-0.19$ and $p=.10$ at 3 months; $\rho=-0.27$ and $p=.03$ at 12 months; and $\rho=-0.05$ and $p=.69$ at 24 months) showed weaker or nonsignificant associations than those between the XQ and PRD scores (Table 2). A comparison of functional assessments of xerostomia and dysphagia, as measured by SSF rates and Swallowing Performance Status Scale scores, also showed weaker or nonsignificant correlations than the correlations between patient-reported assessments (pretreatment: $\rho=0.03$ and $p=.81 ; 3$ months: $\rho=-0.31$ and $p<.01$; 12 months: $\rho=-0.07$ and $p=.58$; and 24 months; $\rho=-0.37$ and $p<.01$; Table 2 ). The negative slopes of these correlations suggested a trend of increasing (worsening) HNQOL-Sw, UWQOL-Sw, and Swallowing Performance Status Scale scores as SSF rates decreased.

\section{Relationship between patient-reported xerostomia and patient-reported dysphagia in patients without and with swallowing impairment of videofluoroscopy}

Among patients with no/mild evidence of swallowing impairment on videofluoroscopy (Swallowing Performance Status Scale score $\leq 3$ ) (Table 3), the posttreatment XQ summary scores remained moderately-to-strongly correlated with the HNQOL-Sw scores at all timepoints ( $\rho=0.50,0.60$, and 0.41 at 3,12 , and 24 months; $p<.01$ for all) and with UWQOL-Sw scores at 3 and 12 months $(\rho=0.55$ and $p<.001$ and $\rho=0.51$ and $p=.002$, respectively; trend at 24 months: $\rho=0.27$ and $p=.10$ ). Among patients with moderate or greater swallowing impairment on videofluoroscopy (Swallowing Performance Status Scale score $\geq 4$ ), similar significant correlations were observed between XQ summary scores and both 


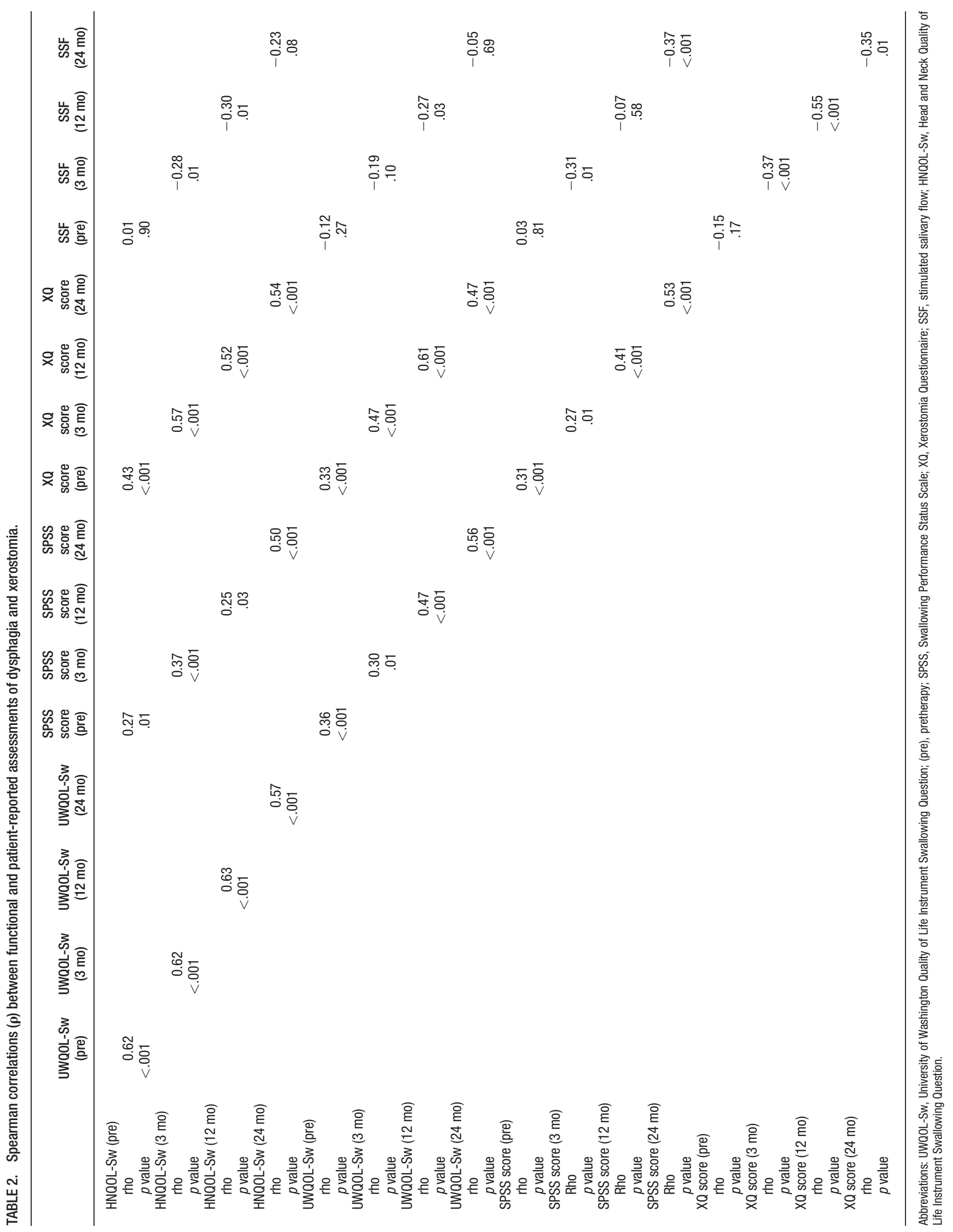


TABLE 3. Patient-reported dysphagia in patients without evidence of significant swallowing impairment on videofluoroscopy.

\begin{tabular}{lcccc}
\hline $\begin{array}{l}\text { Time after } \\
\text { completion of } \\
\text { chemo-IMRT }\end{array}$ & $\begin{array}{c}\text { No. of patients (\%) with } \\
\text { no or mild swallowing } \\
\text { impairment on videofluoroscopy } \\
\text { (SPSS score 1-3) }\end{array}$ & $\begin{array}{c}\text { \% reporting } \geq \text { moderate } \\
\text { bother due to swallowing } \\
\text { problems (HNQOL-Sw) }\end{array}$ & $\begin{array}{c}\text { \% reporting inability } \\
\text { to swallow certain foods } \\
\text { (UWQOL-Sw) }\end{array}$ & $\begin{array}{c}\text { \% reporting swallowing } \\
\text { limited to only soft foods } \\
\text { (UWQOL-Sw) }\end{array}$ \\
\hline 3 mo & $39(44)$ & 21 & 59 & 13 \\
12 mo & $33(41)$ & 12 & 30 & 3 \\
24 mo & $39(60)$ & 5 & 28 & 3
\end{tabular}

Abbreviations: chemo-IMRT, chemotherapy-intensity-modulated radiotherapy; SPSS, Swallowing Performance Status Scale; HNQOL-Sw, Head and Neck Quality of Life Instrument Swallowing Question; UWQOL-Sw, University of Washington Quality of Life Instrument Swallowing Question.

HNQOL-Sw scores $(\rho=0.58,0.32$, and 0.48 at 3,12 , and 24 months; $p<.001, p=.04$, and $p=.02$, respectively) and UWQOL-Sw scores $(\rho=0.32,0.53$, and 0.47 at 3,12 , and 24 months; $p=.03, p<.001$, and $p=.03$, respectively).

\section{Univariate and multivariate regression analyses}

Regression analysis was performed to further assess the relative impact of XQ scores, salivary flow rates, and Swallowing Performance Status Scale scores on posttreatment HNQOL-Sw and UWQOL-Sw scores. At all posttreatment timepoints, both XQ scores and Swallowing Performance Status Scale scores were significantly positively associated on univariate analysis with PRD by both HNQOL-Sw and UWQOL-Sw (Table 4). SSF rates were less associated with HNQOL-Sw and UWQOL-SW scores than were XQ scores, showing significant associations with only HNQOL-Sw at 3 months and 12 months, with trends toward significance at these timepoints for the

TABLE 4. Univariable analysis of association of swallowing impairment of videofluoroscopy, patient-reported xerostomia, and salivary flow with patient-reported dysphagia.

\begin{tabular}{|c|c|c|c|c|}
\hline & Variables & $\begin{array}{c}\text { Normalized } \\
\text { OR }\end{array}$ & $\begin{array}{c}p \\
\text { value }\end{array}$ & $\begin{array}{l}\text { Model } \\
\text { AUC }\end{array}$ \\
\hline \multicolumn{5}{|c|}{ HNQOL-Sw } \\
\hline \multirow[t]{3}{*}{$3 \mathrm{mo}$} & SPSS score & 1.91 & $<.001$ & 0.652 \\
\hline & $X Q$ summary score & 4.03 & $<.001$ & 0.758 \\
\hline & SSF & 0.54 & .035 & 0.630 \\
\hline \multirow[t]{3}{*}{$12 \mathrm{mo}$} & SPSS score & 1.75 & .021 & 0.608 \\
\hline & $X Q$ summary score & 3.77 & $<.001$ & 0.750 \\
\hline & SSF & 0.51 & .03 & 0.649 \\
\hline \multirow[t]{3}{*}{$24 \mathrm{mo}$} & SPSS score & 3.39 & $<.001$ & 0.754 \\
\hline & $X Q$ summary score & 3.12 & $<.001$ & 0.771 \\
\hline & SSF & 0.80 & .37 & 0.608 \\
\hline \multicolumn{5}{|c|}{ UWQOL-SW } \\
\hline \multirow[t]{3}{*}{$3 \mathrm{mo}$} & SPSS score & 1.79 & .005 & 0.643 \\
\hline & $X Q$ summary score & 2.82 & $<.001$ & 0.729 \\
\hline & SSF & 0.58 & .055 & 0.587 \\
\hline \multirow[t]{3}{*}{$12 \mathrm{mo}$} & SPSS score & 3.65 & $<.001$ & 0.742 \\
\hline & $X Q$ summary score & 5.80 & $<.001$ & 0.835 \\
\hline & SSF & 0.60 & .081 & 0.642 \\
\hline \multirow[t]{3}{*}{$24 \mathrm{mo}$} & SPSS score & 4.53 & $<.001$ & 0.795 \\
\hline & $X Q$ summary score & 3.10 & $<.001$ & 0.755 \\
\hline & SSF & 1.01 & .974 & 0.536 \\
\hline
\end{tabular}

Abbreviations: OR, odds ratio; AUC, area under the curve; HNQOL-Sw, Head and Neck Quality of Life Instrument Swallowing Question; SPSS, Swallowing Performance Status Scale; XQ, Xerostomia Questionnaire; SSF, stimulated salivary flow; UWQOL-SW, University of Washington Quality of Life Instrument Swallowing Question.
UWQOL-Sw endpoint and no association at 24 months with either HNQOL-Sw or UWQOL-Sw. On multivariable analysis (Table 5), the XQ scores remained independently associated with both HNQOL-Sw and UWQOL-Sw scores after adjustment for Swallowing Performance Status Scale scores at nearly all timepoints. Moreover, the XQ score was a stronger predictor of HNQOL-Sw and UWQOL-Sw scores than the

TABLE 5. Multivariable analysis of impact of swallowing dysfunction by videofluoroscopy, patient-reported xerostomia, and salivary flow with patient-reported dysphagia.

\begin{tabular}{|c|c|c|c|c|}
\hline $\begin{array}{l}\text { Dysphagia } \\
\text { endpoint }\end{array}$ & Variables & $\begin{array}{c}\text { Normalized } \\
\text { OR }\end{array}$ & $\begin{array}{c}p \\
\text { value }\end{array}$ & $\begin{array}{c}\text { Model } \\
\text { AUC }\end{array}$ \\
\hline \multicolumn{5}{|l|}{ HNQOL-Sw } \\
\hline \multicolumn{5}{|l|}{$3 \mathrm{mo}$} \\
\hline \multirow[t]{2}{*}{ Model 1} & SPSS score & 1.98 & .001 & 0.795 \\
\hline & $\mathrm{XQ}$ summary score & 3.99 & $<.001$ & \\
\hline \multirow[t]{2}{*}{ Model 2} & SPSS score & 2.31 & $<.001$ & 0.708 \\
\hline & SSF & 0.61 & .077 & \\
\hline \multicolumn{5}{|l|}{$12 \mathrm{mo}$} \\
\hline \multirow[t]{2}{*}{ Model 1} & SPSS score & 1.20 & .50 & 0.754 \\
\hline & $\mathrm{XQ}$ summary score & 3.48 & $<.001$ & \\
\hline \multirow[t]{2}{*}{ Model 2} & SPSS score & 1.64 & .058 & 0.702 \\
\hline & SSF & 0.48 & .031 & \\
\hline \multicolumn{5}{|l|}{$24 \mathrm{mo}$} \\
\hline \multirow[t]{2}{*}{ Model 1} & SPSS score & 2.83 & .003 & 0.804 \\
\hline & XQ summary score & 2.05 & .030 & \\
\hline \multirow[t]{2}{*}{ Model 2} & SPSS score & 4.37 & $<.001$ & 0.765 \\
\hline & SSF & 1.11 & .69 & \\
\hline \multicolumn{5}{|l|}{ UWQOL-SW } \\
\hline \multicolumn{5}{|l|}{$3 \mathrm{mo}$} \\
\hline \multirow[t]{2}{*}{ Model 1} & SPSS score & 1.58 & .036 & 0.740 \\
\hline & $\mathrm{XQ}$ summary score & 2.47 & $<.001$ & \\
\hline \multirow[t]{2}{*}{ Model 2} & SPSS score & 2.16 & .001 & 0.704 \\
\hline & SSF & 0.62 & .091 & \\
\hline \multicolumn{5}{|l|}{$12 \mathrm{mo}$} \\
\hline \multirow[t]{2}{*}{ Model 1} & SPSS score & 2.56 & .005 & 0.860 \\
\hline & $\mathrm{XQ}$ summary score & 4.68 & $<.001$ & \\
\hline \multirow[t]{2}{*}{ Model 2} & SPSS score & 3.15 & $<.001$ & 0.758 \\
\hline & SSF & 0.59 & .12 & \\
\hline \multicolumn{5}{|l|}{$24 \mathrm{mo}$} \\
\hline \multirow[t]{2}{*}{ Model 1} & SPSS score & 4.20 & $<.001$ & 0.821 \\
\hline & $\mathrm{XQ}$ summary score & 1.74 & .12 & \\
\hline \multirow[t]{2}{*}{ Model 2} & SPSS score & 7.67 & $<.001$ & 0.842 \\
\hline & SSF & 1.57 & .12 & \\
\hline
\end{tabular}

Abbreviations: OR, odds ratio; AUC, area under the curve; HNQOL-Sw, Head and Neck Quality of Life Instrument Swallowing Question; SPSS, Swallowing Performance Status Scale; XQ, Xerostomia Questionnaire; SSF, stimulated salivary flow; UWQOL-SW, University of Washington Quality of Life Instrument Swallowing Question. 
videofluoroscopy Swallowing Performance Status Scale scores at most timepoints. By contrast, the association between the SSF rates and PRD, after adjustment for videofluoroscopy Swallowing Performance Status Scale score on multivariable analysis, was nonsignificant for nearly all timepoints (Table 5).

\section{DISCUSSION}

This study demonstrates that in patients receiving chemo-IMRT whose planning objectives included the sparing of both salivary and swallowing-related structures, PRX was significantly correlated with PRD. The assessment in the current study of both patient-reported outcomes for dysphagia and xerostomia, as well as the functional assessments of the related physiological dysfunctions (Swallowing Performance Status Scale scores and major salivary gland flow rates), highlights the distinctions in relationships between patient-reported and functional outcome measures, and indirectly suggests potential mechanisms through which xerostomia may affect patient perceptions of dysphagia.

A consistent finding throughout the longitudinal 2-year assessment period was the highly significant relationships of both Swallowing Performance Status Scale scores and XQ scores with PRD. On the other hand, SSF rates, measured from the major salivary glands, had lower correlations with $\mathrm{PRD}$ in the univariate analyses, and were not significant or of only borderline significance in multivariate analysis. Thus, although the functional assessment of swallowing impairment via videofluoroscopy was highly predictive of PRD, patients' subjective perception of xerostomia was an additional highly significant factor.

One of the reasons for the discrepancy between the association of XQ scores and SSF rates on PRD is the weak correlation between xerostomia symptoms and the measured SSF. The only modest correlation coefficients of xerostomia symptoms versus salivary output found in our study posttherapy, despite being statistically significant, suggest that SSF explains only part of the variability in PRX. Although the majority of stimulated saliva during eating is secreted by the parotid glands, these secretions are predominantly serous, consisting almost entirely of water. In contrast, the secretions from the submandibular glands and especially the minor salivary glands dispersed within the oral cavity, although relatively of small volumes, are rich in salivary mucins, which adhere to mucosal and food particle surfaces to provide lubrication for food passage and mucosal protection. Mucins bind water molecules effectively, and their presence on the mucosal surfaces helps maintain these tissues in a hydrated state and, importantly, provide a sense of hydration to the patient. $^{21}$ Reduced salivary mucins after CRT may greatly impact the sensation of dry mouth. ${ }^{30}$ The importance of the mucin-containing secretions is highlighted in the relatively weak correlations between SSF rates, dominated by parotid gland secretions, and PRX in our study. They also explain the failure of randomized studies of parotidsparing IMRT to demonstrate a clinically meaningful advantage in PRX, despite improvements in salivary flow rates and observer-rated xerostomia in the IMRT arm compared with the 2D RT arms. ${ }^{19,28}$ For example, Nutting et $\mathrm{al}^{19}$ reported that through 12 months after treatment, the patient-reported benefit of IMRT versus 2D RT was $<10$ points on a 0 to 100 scale, regarded not clinically relevant, despite a large advantage in salivary output. ${ }^{19}$ Thus, the SSF measurements, which are predominantly a measure of the parotid gland secretions, underestimate the contribution of the mucin-producing glands. This is reflected in studies demonstrating that, in addition to mean parotid gland dose, doses delivered to the submandibular and minor salivary glands affect PRX. ${ }^{29}$ The results of the current study emphasize that their sparing should be an important goal of IMRT optimization, as further improvement in xerostomia is likely to reduce PRD.

Few previous studies have been published assessing the association between PRX and dysphagia in patients with head and neck cancer treated with RT. Logemann et al ${ }^{16}$ measured whole mouth saliva in patients with head and neck cancer from pre-RT to 3 months after RT, and compared salivary output to both PRD and videofluoroscopy results. No significant correlations were found in either this study or in a follow-up study through 12 months, ${ }^{17}$ likely because of the very low salivary output in all patients resulting from 2D-radiation techniques, that precluded meaningful analysis. These authors concluded that reduced saliva did not affect the mechanics of swallowing but seemed to change patients' perception of their swallowing ability. Our study, in which the SSF rates and XQ scores recovered after IMRT, showed significant correlations between XQ scores and SSF rates and dysphagia symptoms even in patients without videofluoroscopy abnormalities. Further support for a causative, rather than merely correlative, relationship between xerostomia and dysphagia is implied by findings in patients with Sjögren's syndrome, who reported worse dysphagia and had prolonged food transit time on videofluoroscopy compared with healthy controls. ${ }^{31}$

The majority of the current literature comparing xerostomia and dysphagia after RT in patients with head and neck cancer had relied upon observer-rated assessments, yielding conflicting results. Some have reported a significant correlation between these toxicities, ${ }^{32}$ whereas others have reported no apparent correlation, ${ }^{33}$ rather showing that significant xerostomia persisted long after therapy compared to prompt improvement in dysphagia. Several retrospective studies comparing parotid-sparing IMRT to 3D-RT noted that, in parallel to improved xerostomia, swallowing was better in the IMRT-treated patients even though no specific effort was made to spare the swallowing-related structures. ${ }^{34-36}$ On the other hand, a randomized study of parotid-sparing IMRT versus 3D-RT found no difference in dysphagia between the arms. ${ }^{19}$ The heterogeneous nature of these studies, which included many patients treated without concurrent chemotherapy, and therefore were at a lesser risk of dysphagia, complicates accurate evaluation of their results.

The observer-rated Radiation Therapy Oncology Group/ European Organisation for Research and Treatment of Cancer scale for dysphagia, which is very similar to the Common Terminology Criteria for Adverse Events evaluation grading system, disclosed very few patients with grades $\geq 2$ at 12 months and beyond, similar to our prior report in patients treated with swallowing-structure- 
sparing IMRT. ${ }^{11}$ The low frequency of ORD events precluded statistical evaluation of correlates with this endpoint. In contrast, patient-reported scores showed a wide distribution. We have previously reported the discrepancies between ORD and PRD in assessing the relative severity of dysphagia, showing that videofluoroscopy results were more consistent with patient-reported than observer-rated scoring. ${ }^{37}$ We therefore prefer using patient-reported outcomes as the most reliable way to assess dysphagia and other sequelae of treatment.

In conclusion, in this longitudinal study of chemoIMRT for patients with oropharyngeal cancer, PRX was a significant contributor to patients' perception of dysphagia, irrespective of evidence of functional swallowing impairment and despite the use of organ-sparing IMRT techniques. Efforts to decrease treatment-related xerostomia by increased salivary gland sparing, including the submandibular and minor oral cavity salivary glands in addition to sparing the parotid glands, ${ }^{25}$ are likely to translate into improvements in patients' perception of dysphagia after CRT for head and neck cancer.

\section{REFERENCES}

1. Ang KK, Harris J, Wheeler R, et al. Human papillomavirus and survival of patients with oropharyngeal cancer. N Engl J Med 2010;363:24-35.

2. Hassan SJ, Weymuller EA Jr. Assessment of quality of life in head and neck cancer patients. Head Neck 1993;15:485-496.

3. Hunter KU, Schipper M, Feng FY, et al. Toxicities affecting quality of life after chemo-IMRT of oropharyngeal cancer: prospective study of patientreported, observer-rated, and objective outcomes. Int J Radiat Oncol Biol Phys 2013;85:935-940.

4. Mittal BB, Pauloski BR, Haraf DJ, et al. Swallowing dysfunction-preventative and rehabilitation strategies in patients with head-and-neck cancers treated with surgery, radiotherapy, and chemotherapy: a critical review. Int J Radiat Oncol Biol Phys 2003;57:1219-1230.

5. Eisbruch A, Schwartz M, Rasch C, et al. Dysphagia and aspiration after chemoradiotherapy for head-and-neck cancer: which anatomic structures are affected and can they be spared by IMRT? Int J Radiat Oncol Biol Phys 2004;60:1425-1439.

6. Hunter KU, Lee OE, Lyden TH, et al. Aspiration pneumonia after chemointensity-modulated radiation therapy of oropharyngeal carcinoma and its clinical and dysphagia-related predictors. Head Neck 2014;36:120-125.

7. Logemann JA. The role of exercise programs for dysphagia patients. Dysphagia 2005;20:139-140.

8. Feng FY, Kim HM, Lyden TH, et al. Intensity-modulated radiotherapy of head and neck cancer aiming to reduce dysphagia: early dose-effect relationships for the swallowing structures. Int J Radiat Oncol Biol Phys 2007; 68:1289-1298.

9. Dirix P, Abbeel S, Vanstraelen B, Hermans R, Nuyts S. Dysphagia after chemoradiotherapy for head-and-neck squamous cell carcinoma: doseeffect relationships for the swallowing structures. Int J Radiat Oncol Biol Phys 2009; 75:385-392.

10. Eisbruch A, Kim HM, Feng FY, et al. Chemo-IMRT of oropharyngeal cancer aiming to reduce dysphagia: swallowing organs late complication probabilities and dosimetric correlates. Int J Radiat Oncol Biol Phys 2011;81: e93-e99.

11. Feng FY, Kim HM, Lyden TH, et al. Intensity-modulated chemoradiotherapy aiming to reduce dysphagia in patients with oropharyngeal cancer: clinical and functional results. J Clin Oncol 2010;28:2732-2738.

12. Popovtzer A, Cao Y, Feng FY, Eisbruch A. Anatomical changes in the pharyngeal constrictors after chemo-irradiation of head and neck cancer and their dose-effect relationships: MRI-based study. Radiother Oncol 2009; 93:510-515

13. Kendall KA, Kosek SR, Tanner K. Quality-of-life scores compared to objective measures of swallowing after oropharyngeal chemoradiation. Laryngoscope 2014;124:682-687.

14. Rogus-Pulia NM, Pierce MC, Mittal BB, Zecker SG, Logemann JA. Changes in swallowing physiology and patient perception of swallowing function following chemoradiation for head and neck cancer. Dysphagia 2014;29:223-233.
15. Ding R, Logemann JA. Patient self-perceptions of swallowing difficulties as compared to expert ratings of videofluorographic studies. Folia Phoniatr Logop 2008;60:142-150.

16. Logemann JA, Smith $\mathrm{CH}$, Pauloski BR, et al. Effects of xerostomia on perception and performance of swallow function. Head Neck 2001;23:317-321.

17. Logemann JA, Pauloski BR, Rademaker AW, et al. Xerostomia: 12-month changes in saliva production and its relationship to perception and performance of swallow function, oral intake, and diet after chemoradiation. Head Neck 2003;25:432-437.

18. Eisbruch A, Kim HM, Terrell JE, Marsh LH, Dawson LA, Ship JA. Xerostomia and its predictors following parotid-sparing irradiation of head-andneck cancer. Int J Radiat Oncol Biol Phys 2001;50:695-704.

19. Nutting CM, Morden JP, Harrington KJ, et al. Parotid-sparing intensity modulated versus conventional radiotherapy in head and neck cancer (PARSPORT): a phase 3 multicentre randomised controlled trial. Lancet Oncol 2011;12:127-136.

20. Hunter KU, Fernandes LL, Vineberg KA, et al. Parotid glands dose-effect relationships based on their actually delivered doses: implications for adaptive replanning in radiation therapy of head-and-neck cancer. Int $J$ Radiat Oncol Biol Phys 2013;87:676-682.

21. Murdoch-Kinch CA, Kim HM, Vineberg KA, Ship JA, Eisbruch A. Doseeffect relationships for the submandibular salivary glands and implications for their sparing by intensity modulated radiotherapy. Int J Radiat Oncol Biol Phys 2008;72:373-382.

22. Terrell JE, Nanavati KA, Esclamado RM, Bishop JK, Bradford CR, Wolf GT. Head and neck cancer-specific quality of life: instrument validation. Arch Otolaryngol Head Neck Surg 1997;123:1125-1132.

23. Cox JD, Stetz J, Pajak TF. Toxicity criteria of the Radiation Therapy Oncology Group (RTOG) and the European Organization for Research and Treatment of Cancer (EORTC). Int J Radiat Oncol Biol Phys 1995;31: 1341-1346.

24. Meirovitz A, Murdoch-Kinch CA, Schipper M, Pan C, Eisbruch A. Grading xerostomia by physicians or by patients after intensity-modulated radiotherapy of head-and-neck cancer. Int J Radiat Oncol Biol Phys 2006;66: 445-453.

25. Karnell MP, MacCracken E. A database information storage and reporting system for videofluorographic oropharyngeal motility (OPM) swallowing evaluations. Am J Speech Lang Pathol 1994;8:54-60.

26. Salama JK, Stenson KM, List MA, et al. Characteristics associated with swallowing changes after concurrent chemotherapy and radiotherapy in patients with head and neck cancer. Arch Otolaryngol Head Neck Surg 2008;134:1060-1065.

27. Stenson KM, MacCracken E, List M, et al. Swallowing function in patients with head and neck cancer prior to treatment. Arch Otolaryngol Head Neck Surg 2000;126:371-377.

28. Karnell M, Maccracken E, Moran W, Vokes E, Haraf D, Panje W. Swallowing function following multispecialty organ preservation treatment of advanced head and neck-cancer. Oncol Rep 1994;1:597-601.

29. Little M, Schipper M, Feng FY, et al. Reducing xerostomia after chemoIMRT for head-and-neck cancer: beyond sparing the parotid glands. Int $J$ Radiat Oncol Biol Phys 2012;83:1007-1014.

30. Randall K, Stevens J, Yepes JF, et al. Analysis of factors influencing the development of xerostomia during intensity-modulated radiotherapy. Oral Surg Oral Med Oral Pathol Oral Radiol 2013;115:772-779.

31. Rhodus NL, Colby S, Moller K, Bereuter J. Quantitative assessment of dysphagia in patients with primary and secondary Sjögren's syndrome. Oral Surg Oral Med Oral Pathol Oral Radiol Endod 1995;79:305-310.

32. Mortensen HR, Jensen K, Aksglaede K, Behrens M, Grau C. Late dysphagia after IMRT for head and neck cancer and correlation with dose-volume parameters. Radiother Oncol 2013;107:288-294.

33. Al-Mamgani A, Van Rooij P, Tans L, Teguh DN, Levendag PC. Toxicity and outcome of intensity-modulated radiotherapy versus 3-dimensional conformal radiotherapy for oropharyngeal cancer: a matched-pair analysis. Technol Cancer Res Treat 2013;12:123-130.

34. Vergeer MR, Doornaert PA, Rietveld DH, Leemans CR, Slotman BJ, Langendijk JA. Intensity-modulated radiotherapy reduces radiationinduced morbidity and improves health-related quality of life: results of a nonrandomized prospective study using a standardized follow-up program. Int J Radiat Oncol Biol Phys 2009;74:1-8.

35. Modesto A, Laprie A, Vieillevigne L, et al. Intensity-modulated radiotherapy for laryngeal and hypopharyngeal cancer: minimization of late dysphagia without jeopardizing tumor control. Strahlenther Onkol 2015;191:225-233.

36. Pauloski BR, Rademaker AW, Logemann JA, Discekici-Harris M, Mittal BB. Comparison of swallowing function after intensity-modulated radiation therapy and conventional radiotherapy for head and neck cancer. Head Neck 2015;37:1575-1582.

37. Gluck I, Feng FY, Lyden T, et al. Evaluating and reporting dysphagia in trials of chemoirradiation for head-and-neck cancer. Int J Radiat Oncol Biol Phys 2010;77:727-733. 